\title{
PERIOPERATIVE NUTRITION IN PEDIATRIC SURGICAL PATIENTS
}

\author{
K HASINA ${ }^{1}$, SMS ENAYET ${ }^{2}$, A HANIF $^{3}$
}

\section{Introduction:}

Perioperative nutritional care for neonates, infants and younger children differ greatly from those seen in older children and adults. Nutritional care is essential for accelerated growth and development along with homeostasis and postoperative healing of pediatric surgical patients. Depletion of body stores decreased immunocompetence and increased mortality and morbidity is frequently associated with hyper metabolic state. Abdominal surgery is the most stressful factor leading to the reorganization of metabolic processes, redox homeostasis and immune changes. ${ }^{1}$ About $18 \%$ to $40 \%$ of pediatric surgical patients have malnutrition. ${ }^{2}$ Patients at risk for malnutrition are surgical patients, patients with large open wounds (concomitant loss of protein and increased metabolic needs), extensive burns, blunt trauma and sepsis. ${ }^{2}$ Individualized, adequate nutritional support in the peri-operative period decreases morbidity and mortality. ${ }^{3}$ Over past few decades mortality associated with surgery in children has rapidly declined. Refined respiratory care, antibiotics and improved nutrition with a greater knowledge of the needs of the surgical patients have together ameliorated the chances for surgical neonates and infants. ${ }^{4}$

\section{Metabolic response:}

The acute metabolic response represents a catabolic state. Metabolic response during the catabolic phase leads to decrease in tissue stores of protein, carbohydrate, and fat resulting in failure to thrive. Table

1. Dr. Kaniz Hasina, Assoc Professor, Dept of Pediatric Surgery, Dhaka Medical College \& Hospital

2. Dr. SM Sabbir Enayet, Resident, Dept of Pediatric Surgery, Dhaka Medical College \& Hospital

3. Dr. Abdul Hanif, Professor, Dept of Pediatric Surgery, Dhaka Medical College \& Hospital

Correspondence to : Dr. Kaniz Hasina, Assoc Professor, Dept of Pediatric Surgery, Dhaka Medical College \& Hospital. E-mail: kanizhasina@gmail.com
1 shows the metabolic status in critically ill patients. In the postoperative period, because of the reductions in activity and insensible losses observed in sedated infants in a thermo-neutral intensive care environment, caloric requirements are reduced to amounts necessary to meet basal metabolic needs. Though historically, it has been thought that the surgical patient requires an energy intake proportional to the severity of the illness recent evidence reveals that the increase in energy expenditure associated with surgery only lasts for 24 hours after the procedure. ${ }^{5}$ So estimating energy expenditure during the first 24 hours after surgery will overestimate the energy requirements for the entire postoperative period, potentially resulting in excess energy intake with its potential consequences. ${ }^{6}$

Table-I

Metabolic status in critically ill infants

\begin{tabular}{|c|c|}
\hline Energy & $\begin{array}{l}\text { hypermetabolic state } \\
\uparrow O 2 \text { consumption } \\
\text { maldistribution of blood flow } \\
\uparrow \text { gluconeogenesis }\end{array}$ \\
\hline Carbohydrate & $\begin{array}{l}\text { Hyperglycemia } \\
\uparrow \text { respiratory quotient (initially) } \\
\text { 个pyruvate and lactate } \\
\text { insulin resistance }\end{array}$ \\
\hline Protein & $\begin{array}{l}\text { Tprotein catabolism } \\
\text { } \text { gluconeogenicaminoacids (via Coricycle) } \\
\text { } \text { glutamine production (gut mucosal fuel) } \\
\downarrow \text { hemoglobin synthesis } \\
\text { 个inflammatory mediators } \\
\downarrow \text { ! albumin synthesis }\end{array}$ \\
\hline Fat & $\begin{array}{l}\text { } \text { lipolysis } \\
\downarrow \text { lipogenesis } \\
\uparrow \text { triglyceride oxidation } \\
\downarrow \text { ketone bodies }\end{array}$ \\
\hline
\end{tabular}




\section{Nutritional Assessment:}

Despite progress and the development of new techniques to assess nutritional status, assessment still remains complicated for the pediatric surgical patient, and a reliable assessment of nutritional status depends on various parameters. This fact makes the nutritional assessment hard and laborious, but essential for the institution of an adequate nutritional therapy.

Assessment of nutritional status consists of subjective and objective components.

a. Subjective assessment-

Two basic tools

- Mini Nutrition Assessment

- Subjective Global Assessment (SGA).

b. Objective assessment-

- Basic anthropometric measurements

- Height

- Weight

- Head circumference.

It is important to note that despite the recent advances in nutritional assessment, a clinical evaluation of the patient's status (subjective global assessment) performed by a experienced physician can be as reliable as more sophisticated tests. However, this subjective global assessment is better applied to adult patients than to pediatric patients.

Subjective Global Assessment (SGA) is an accurate mode of assessing malnutrition for both inpatients as well as non hospitalized patients. It is performed during the history and physical examination. SGA should include -evaluation of weight loss (5\% for mild to $10 \%$ for moderate to severe malnutrition), anorexia, vomiting, physical evidence of muscle wasting (indicative of severe malnutrition)

In basic anthropometric measurements, values are placed on a standardized growth curve and expected weight for height index is calculated. Body mass index (BMI) is used for $>2$ years of age. Length is an excellent indicator of long-term body growth. Length and head circumference is less affected by excess fat or postoperative fluid fluctuations.

Serum albumin ( $t 1 / 2$ of 20 days) is seen to assess chronic nutritional status. Other parameters for nutritional assessment are bone age and dental status.

\section{Energy requirement:}

Energy needs of infants and children are unique. Nutritional reserve in a 1-kg premature infant is for 4 days, whereas in a full-term infant it is about 1 month In children, energy is required for maintenance of body metabolism and growth. Calorie requirements (Kcal/ $\mathrm{kg} /$ day) should be matched to the amount of fluid needs ( $\mathrm{ml} / \mathrm{kg} / \mathrm{day})$. Table 2 shows daily fluid requirements and table 3 shows daily calorie requirement in pediatric age group.

Table-II

Daily Fluid Requirements for Pediatric Patients

\begin{tabular}{ll}
\hline Body Weight & Amount \\
\hline$<1500 \mathrm{~g}$ & $130-150 \mathrm{mlAg}$ \\
$1500-2000 \mathrm{~g}$ & $110-130 \mathrm{mlAg}$ \\
$2-10 \mathrm{~kg}$ & $100 \mathrm{mlAg}$ \\
$>10-20 \mathrm{~kg}$ & $1000 \mathrm{~mL}$ for $10 \mathrm{~kg}+50 \mathrm{~mL} / \mathrm{kg}$ \\
& for each $\mathrm{kg}>10$ \\
$>20 \mathrm{~kg}$ & $1500 \mathrm{ml}$ for $20 \mathrm{~kg}+20 \mathrm{~mL} / \mathrm{kg}$ \\
& for each $\mathrm{kg}>20$ \\
\hline
\end{tabular}

Table-III

Energy Requirements

Daily Energy Requirements (Total kcal/kg) for Pediatric Patients

\begin{tabular}{lc}
\hline Preterm neonate & $90-120$ \\
$<6$ months & $85-105$ \\
$6-12$ months & $80-100$ \\
$1-7$ years & $75-90$ \\
$7-12$ years & $50-75$ \\
$>12-18$ years & $30-50$ \\
\hline
\end{tabular}

\section{Nutritional Delivery system:}

Enteral Nutrition (EN) includes oral nutritional supplementation and tube feedings either nasogastric or nasojejunal. For patients requiring feedings for more than 8 weeks, a more permanent feeding access (e.g., gastrostomy tube) should be considered. EN should be the primary source of nutrients if the gastrointestinal tract is functional. Infants in a state of good health before surgery or trauma can sustain 5 to 7 days without significant energy intake and without serious systemic consequences, provided that adequate nutritional support is initiated thereafter. Enteral feedings are begun after the resolution of the postoperative ileus.

Parenteral Nutrition (PN) is the intravenous administration of balanced and complete nutrition to support anabolism, prevent weight loss, or promote 
weight gain. It should be used for the shortest time possible. Indications for PN are inability to tolerate enteral feedings, gastrointestinal disorders (shortbowel syndrome, malabsorption, intractable diarrhea, bowel obstruction, protracted vomiting, inflammatory bowel disease, enterocutaneous fistulas), congenital anomalies (gastroschisis, bowel atresia, volvulus, meconium ileus), radiation therapy to the gastrointestinal tract, chemotherapy resulting in gastrointestinal dysfunction, severe respiratory distress syndrome in premature infants, Very-low-birthweight infants - during the first 24 hours following birth, young infants- $>4$ to 5 days starvation, children and adults - $>7$ to 10 days starvation .

\section{Nutritional support in pediatric surgical patients:}

The pediatric surgical patient responds to the stress of surgery quite differently than older children or adults. Operative stress (like induction of anesthesia) markedly affects metabolism in children, such as fentanyl have a beneficial effect in reducing the catabolic effect. In neonates protein turnover and catabolism seems not to be affected by major operative procedures and parenteral nutrition is associated with increased production of oxygen-free radicals leading to immunosuppression.

Preoperative $\mathrm{PN}$ in mild to moderate malnutrition has little benefit and more complications. But in severe malnutrition it has significant benefits. The use of PN may actually predispose patients to increased infectious complications. Delay in operative management in order to provide preoperative $\mathrm{PN}$ is not indicated except in severe malnutrition.

Postoperative nutrition should be started early, using enteral nutrition (EN) or a combination of PN and EN until the gastrointestinal tract fully recovers. Postoperative PN has positive effect on nitrogen balance and levels of insulin growth factor-1(IGF-I) though there is no clinical benefit with negligible effect on postoperative healing and higher infection rates. Postoperative PN should be restricted to infants who will not tolerate short period of starvation, older children who will probably not start enteral nutrition for at least 5 to 7 days. In well-nourished adolescents, this period of time should increase to 7 to 10 days.

Critically III Surgical patients manifest with poor enteral feeding, anorexia, paralytic ileus (often), insulin resistance results in hyperglycemia and hypertriglyceridemia and progressively reduced visceral protein stores. Energy needs of postoperative or septic critically ill infants are commonly overestimated. During periods of sepsis and critical illness there is cessation of growth and marked decrease in energy needs. So almost one third of an infant's energy needs is provided to support growth ( 30 to $35 \mathrm{kcal} / \mathrm{kg} /$ day).

In an infant with biliary atresia after a clinically successful hepatic portoenterostomy, bile flow into the intestine is reduced causing profound defect in fat digestion and absorption, deficiency of essential fatty acid, inadequate absorption of fat-soluble vitamins, consequently, lack of bone mineralization \& failure to thrive. So nutrition in patients with biliary atresia should provide adequate calories using a formula that maximizes fat intake. This formula should have large amount of medium-chain triglycerides and sufficient linoleic acid to prevent fatty acid deficiency in the face of decreased absorption. When PN is needed, standard crystalline amino acid solution should be used. Breastfeeding should be used cautiously due to high fat content. Vitamin supplementation is critical in patients with biliary atresia.

In short bowel syndrome initially main or sole caloric source will be through PN, enteral feedings should be initiated as soon as possible. Enteral feedings stimulate small-bowel adaptation and prevent the development of PN associated cholestasis. Ideal enteral solution should be isotonic. The protein source should be predominately elemental.

\section{Monitoring:}

Monitoring of nutritional status in a pediatric surgical patient is particularly mandatory during the first few days of PN specially TPN. Monitoring of nutritional status is summarized in table $4 .{ }^{5}$

Table-IV

Monitoring of nutritional status

\begin{tabular}{llll}
\hline Daily & Weekly & 2-3x/week & Biweekly \\
\hline Fluid input and output & Height & Serum glucose & Triglycerides \\
Body weight & Head circumference & (reagent strip) & Albumin \\
& Arm circumference & Glucosuria & Pre-albumin \\
& Triceps skinfold Nitrogen & Serum electrolytes & Indirect calorimetry \\
& balance - if available & & - if available \\
\hline
\end{tabular}




\section{Final Considerations:}

a. In any surgical patient nutritional status should be assessed by methods described already. In elective cases if the nutritional status is not optimum it should be corrected preoperatively. In emergency cases there no scope of delay. Surgery should be done with special care regarding nutritional improvement in per and post operative period.

b. In chronically ill children nutritional status should be improved as close to normal as possible without causing undue delay in surgical intervention.

c. Prolonged preoperative fasting is not beneficial as it may cause hypovolemia, hypoglycaemia, dehydration, discomfort, irritability, increased catabolic response to trauma, impaired immunity, and increased post operative insulin resistance.

d. Most surgeons prefer early commencement of oral feeding though the decision is mostly case sensitive.

e. TPN should be considered in case of an anticipated delay of $>4$ days before adequate enteral feeding may be tolerated( Surgery for intestinal atresia, meconium ileus, gastroschisis, necrotising enterocolitis, short bowel syndrome, high output intestinal fistula), in premature babies and in babies with major cardiopulmonary abnormalities ( Congenital Diaphragmatic Hernia).

f. TPN is not possible with our available resources in children. But parenteral nutrition with oral supplements can be easily managed.

\section{References:}

1. Shumilov PV, Netrebenko OK, Mayorova OA, Mukhina YG. Postoperative nutrition in children undergoing abdominal surgery. J Pediatr Gastroenterol Nutr 2004;39:p S492.

2. Teitelbaum DH, Btaiche IF, Coran AG. Nutritional support in pediatric surgical patient. In: Coran AG, Adzick NS, Krummel TM, Laberge JM, Shamberger RC, Caldamone AA, editors. Pediatric Surgery. $7^{\text {th }}$ ed, vol.1, Philadelphia : Elsevier Saunders; 2012. P. 179-199.

3. Chwals WJ. Metabolism and nutritional frontiers in pediatric surgical patients. Surg Clin North Am 1992; 72:1237-1266.

4. Teitelbaum DH, Coran AG. Perioperative nutritional support in pediatrics. Nutrition 1998;14: 130-42.

5. Lloyd DA. Energy requirements of surgical newborn infants receiving parenteral nutrition. Nutrition 1998; 14:101-04.

6. Shulman RJ, Phillips S. Parenteral nutrition in infants and children. J Pediatr Gastroenterol Nutr 2003; 36:587-607. 